\title{
An Agent-Based Approach for Evaluating Basic Design Options of Management Accounting Systems
}

\author{
Friederike Wall, Alpen-Adria-Universität Klagenfurt, Austria
}

\begin{abstract}
This paper investigates the effectiveness of reducing errors in management accounting systems with respect to organizational performance. In particular, different basic design options of management accounting systems of how to improve the information base by measurements of actual values are analyzed in different organizational contexts. The paper applies an agent-based simulation based on the idea of NK fitness landscapes. The results provide broad, but no universal support for conventional wisdom that lower inaccuracies of accounting information lead to more effective adaptation processes. Furthermore, results indicate that the effectiveness of improving the management accounting system subtly interferes with the complexity of the interactions within the organization and the coordination mode applied.
\end{abstract}

Keywords - Agent-based Simulation; Complexity; Coordination; Learning; Management Accounting Systems

\section{INTRODUCTION}

$\mathbf{M}^{2}$ ANAGEMENT ACCOUNTING is intended to provide decision-makers with judgmental information for evaluating options and to produce information for assessing managerial performance [1], [2].. For deciding whether, or not, to change the status quo in favor of an alternative option, a decision-maker requires information on the pay-offs of both options. Information related to the status quo may result from measurements of actual values (i.e., "weighting", "counting" and valuing) within accounting systems; and unfortunately, it cannot be taken for granted that these measurements perfectly reflect reality [3]. The alternative options, in principle, are subject to ex ante-evaluations by decision-makers who, according to Simon [4] may suffer from cognitive limitations. However, also ex ante-evaluations might be based on measurements, i.e., actual values received on basis of decisions made in former periods and used to "learn" for future decisions. For instance, plan cost accounting often relies on cost functions which are built from actual costs realized in former periods [5] - or as Christensen\} [3] puts it: "[o]nly autocorrelation makes historical accounting relevant for decision purposes" (p. 1827).

Moreover, management accounting systems are embedded in an organizational structure and the organizational structure affects imperfections of judgmental information. In particular, in organizations the overall decision problem is segmented into partial decisions which are delegated to decentral decision-makers (e.g., [6]-[8]). With delegation further difficulties occur: partial decisions may be interdependent, decision-makers likely have different information and pursue their own objectives opportunistically. To avoid losses with respect to the organization's performance, coordination is required, though, according to Ackoff [9], more intense coordination not necessarily increases organizational performance.

Against this background the paper investigates the following research question: In which settings of organizational structure and basic design options of the management accounting system it is effective to use measured actual values by management accounting systems for improving judgmental information?

Hence, the paper focuses on imperfect knowledge of pay-off functions in organizations. The paper does not address decision-making under uncertainty due to imperfectly known future events [10]. Furthermore, the paper does not consider the diverse biases and heuristics that individuals suffer from in case of uncertainty [11]. We regard accounting errors in terms of noise as the difference between estimated and correct values [12]. [13]; however, the paper does not relate to biases in accounting in terms of the application of accounting principles that is not in line with the accounting principles.

For investigating the research question, a method is required that allows controlling a multitude of issues in interaction with each other like interdependent decisions, coordination mechanisms, inaccuracies of judgmental information and related adjustments due to measurement of actual values by accounting. Obviously, these interrelated issues would be particularly difficult to control in empirical research and would induce intractable dimensions in formal modeling. In contrast, simulation methods allow dealing with manifold interdependent issues [14]. Since the research question focuses on collaborative decision-making an agent-based simulation appears appropriate.

The paper contributes to research since, to the best of the author's knowledge, for the first time different settings of memorizing actual values and dynamic adjustments through 
actual values in management accounting are investigated in interaction with major organizational design variables. Moreover, using an agent-based method is a relatively new approach in the area of management accounting ([15)-[18]).

The remainder of this article is organized as follows: Section II places the research question within the context of related literature. In the third chapter we introduce the simulation model and in Section IV we present and discuss results of the simulations.

\section{RELATED LITERATURE IN ACCOUNTING AND ORGANIZATION SCIENCE}

The research question of this article obviously refers to the body of research on errors in accounting. However, our study might also be seen in the context of research on a more general question: how does organizational design influence the overall outcome of an organization with decision-makers imperfectly informed about the outcome of alternatives? Subsequently, we outline these streams of research with respect to the research question addressed in this paper.

\section{A. Errors in Accounting}

Christensen [3] gives a recent overview and discussion on errors in accounting. Stating that errors in accounting are "often neglected when the design of accounting systems is evaluated" (p. 1836) he elaborates three dimensions of this subject. Firstly, accounting information serves to update expectations of future events of the firm (e.g., future costs, cash flows). In this sense accounting is a source of learning and, in particular, allows updating beliefs. Thus, the main question is whether the accuracy of information known beforehand and the accounting information leads to more reliable expectations about future events.

Secondly, Christensen [3] points out that particularly cost accounting in various contexts is based on linear cost functions and that linearity does not necessarily reflect reality perfectly. Thus, the accounting system suffers from an endogenous error. This line of argumentation also relates to the findings of Datar and Gupta [19] who analyze the effects of erroneous choices of cost drivers in product costing and to the findings of Labro and Vanhoucke [20] related to the interactions among errors in activity based costing. Recently, Leitner [16], [17] investigates interactions among errors and biases in traditional costing systems.

Thirdly, Christensen [3] states that accounting information not necessarily is the best sort of information for a certain purpose and that, for example, the price mechanism might reveal better condensed information. In this sense, applying accounting systems rather than, for example, the market mechanism is the erroneous choice.

\section{B. Imperfect Information on Pay-Off in Organizations}

The seminal work of Sah and Stiglitz [21]-[23] may be regarded as the starting point of the stream of research which investigates the robustness of different organizational structures against so-called type I and type II errors: In analogy to statistical inference, imperfect information used in decision-making basically can lead to two different types of errors: in case of "type I errors" an option that, in fact, is superior compared to the status quo is rejected due to a false negative ex ante-evaluation. In contrary, with "type II errors" a false positive option is chosen since it is perceived to be superior to the status quo, whereas, in fact, it is inferior.

In their 1986 paper Sah and Stiglitz [22] introduce a project-selection-framework: An organization consists of several decision-making units which receive knowledge of feasible projects. Imperfect ex ante-evaluations could occur in case that a "good" project which, in fact, would increase organizational performance is rejected (type I error) or if a "bad" project which, in fact, reduces organizational performance is accepted (type II error). Each decision-making unit is characterized by a screening function. The screening function gives the probability that a project is accepted as a function of the project's quality, i.e., the project's contribution to performance.

Sah and Stiglitz [22] distinguish two "architectures" of the decision-making organization: polyarchy and hierarchy. In the polyarchy, each decision-maker can decide in favor of a project independent from other decision-makers. In the hierarchy, in case that a decision-maker on a lower level positively evaluates a project, the project proposal has to be forwarded to a decision-making unit of a higher level. Thus, for acceptance in a two-level hierarchy, a project has to be positively evaluated twice. Sah and Stiglitz [22] show that the hierarchy reduces the likelihood that projects are accepted which better should have been rejected, i.e., hierarchies reduce type II errors; in contrary, in a polyarchic structure the tendency to falsely reject "good" projects, i.e., the occurrence of type I errors, is reduced.

The works of Sah and Stiglitz initiated further research on the decision-making properties of hierarchical versus polyarchic organizations. For example, Koh [24], [25] introduces costs for information gathering and processing on the decision-makers site and information asymmetries related to the decision-makers actions into the project-selectionframework. In the study of Visser [26] the decision-making units do not suffer from errors in their judgments but rather from obstacles to fully communicate the information they have to other decision-making units. Christensen and Knudsen [27] extend the work of Sah and Stiglitz [21]-[23] by investigating the range of organizational structures between polyarchy and hierarchy and provide a general framework for designing decision-making structures that most effectively reduce type-I and type-II errors.

It is worth mentioning that some aspects of complex decentralized decision-making systems might differ from the project-selection-framework of Sah and Stiglitz: the projects under evaluation are independent from each other, i.e., no interactions between the single project options (or decisions) exist. However, there are also decision problems which cannot 
be segmented without inducing interactions among partial decisions and, to some extent, interactions are a consequence of specialization. The approach presented subsequently takes segmented decisions with interactions among partial decisions into account.

\section{SIMULATION MODEL}

The simulation model is based on the NK model introduced by Kauffman [28], [29] of evolutionary biology and successfully applied in management research (e.g. [30]-[32], for an overview [33]). The NK model allows representing a multi-dimensional decision problem where $N$ denotes the number of dimensions and $K$ the level of interactions among these dimensions. However, so far the NK model has rarely been employed to analyze decision-making with imperfect judgmental information [15], [18], [34].

We adopt an advanced version of the NK model with noisy fitness landscapes, as introduced by Levitan and Kauffman [34]. In particular, to analyze our research question the model consists of three components which are presented in the subsequent sections: (1) the organizational structure which is mapped similar to Siggelkow and Rivkin [31]; (2) a representation of imperfect judgmental information that corresponds to organizational segmentation and specialization; (3) alternative modes of how inaccuracies in judgmental information might be reduced in the course of the adaptive walks by measurements by the management accounting system. Thus, the components (2) und (3) are regarded to be the distinctive features of the model.

\section{A. Organizational Structure}

In each time step $t$ of the overall observation period $T$ the artificial organizations face an $\mathrm{N}$-dimensional binary decision problem $\mathbf{d}_{t}=\left(d_{t, 1}, \ldots d_{t, \mathrm{~N}}\right)$, i.e., they have to make decisions $d_{t, i}$ $\in\{0,1\}$ and $i=1, \ldots N$. Each single state of decision $d_{t, i}$ provides a contribution $C_{t, i}$ with $0 \leq C_{t, i} \leq 1$ to organizational performance $\mathrm{V}\left(\mathbf{d}_{t}\right)$. A decision $d_{t, i}$ might interact with $K$ other decisions (for simplicity $K$ assumed to be stable over time). Hence, $K$ can take values from 0 (no interactions) to $\mathrm{N}-1$ (maximum interactions). Thus, performance contribution $C_{t, i}$ may not only depend on the single decision $d_{t, i}$ but also on $K$ other decisions so that

$$
C_{t, i}=f_{i}\left(d_{t, i}, d_{t, i}^{1}, \ldots, d_{t, i}^{K}\right)
$$

In line with the NK model, we assume that for each possible vector $d_{t, i}, d_{t, i}^{1}, \ldots, d_{t, i}^{K}$ the value of $C_{t, i}$ is randomly drawn from a uniform distribution over the unit interval, i.e., $\mathrm{U}[0,1]$. Hence, given equation 1, whenever one of the states $d_{t, i}, d_{t, i}^{1}, \ldots, d_{t, i}^{K}$ of the single decisions is altered, another (randomly chosen) performance contribution $C_{t, i}$ becomes effective. The overall performance $V\left(\mathbf{d}_{t}\right)$ is given as normalized sum of performance contributions $C_{t, i}$ with

$$
V\left(\mathbf{d}_{t}\right)=\frac{1}{N} \sum_{i=1}^{N} C_{t, i}=\frac{1}{N} \sum_{i=1}^{N} f_{i}\left(d_{t, i}, d_{t, i}^{1}, \ldots, d_{t, i}^{K}\right)
$$

Our organizations consist of a main office and $R$ departments subscripted by $r$. Each department has a department head. Our organizations segment their $\mathrm{N}$ dimensional decision problem $\mathbf{d}$ into $R$ disjoint partial problems and delegate each partial problem to one of the $R$ departments. Hence, each department has primary control over a subset of the $N$ single decisions $d_{t, i}$ (e.g., in case of $N=10$ and $R=3$ department 1 over decisions 1 to 3 , department 2 over decisions 4 to 7 and department 3 over decisions 8 to 10), and from the perspective of a certain department $r$ the organizational decision problem is partitioned into a partial decision vector $\mathbf{d}_{t, r}^{\text {own }}$ related to those single decisions which are in the "own" responsibility and into $\mathbf{d}_{t, r}^{\text {res }}$ for the "residual" decisions that other departments are in charge of. However, in case of cross-departmental interactions, choices of a certain department may affect the contributions of decisions other departments are in charge of and vice versa.

In each period $t$ of the adaptive walk a department head seeks to identify the best configuration for the "own" subset of choices assuming that the other departments $q=1, \ldots R$ and $q \neq r$ do not alter their prior subsets $\mathbf{d}_{t-1, q}^{* \text { own }}$ of decisions. In each time period a department head randomly discovers two alternative partial configurations of those binary decisions that he/she is in charge of: an alternative configuration $\mathbf{d}_{t, r}^{a 1}$ that differs in one decision (a1) and another alternative $\mathbf{d}_{t, r}^{a 2}$ which differs in two decisions (a2) compared to the status quo, i.e., $\mathbf{d}_{t-1, r}^{* \text { own }}$. In each time period department head has three options to choose from, i.e., keeping the status quo $\mathbf{d}_{t-1, r}^{* \text { own }}$ and the two alternatives $\mathbf{d}_{t, r}^{a 1}, \mathbf{d}_{t, r}^{a 2}$. According to economic literature, a department head favors that option which he/she perceives to promise the highest value base for compensation. In our model department heads are compensated on basis of the overall performance of the organization according to a linear incentive scheme so that we can ignore conflicts of interests between the organizational and departmental objectives.

However, due to specialization our department heads have different knowledge about the organization's decision problem $\mathbf{d}_{t}$ (we return to that point in the section III.B). In consequence, even though in our model no conflicts of interests occur, departments can have different preferences which might evoke a need for coordination. We analyze two different modes of coordination (for these and other modes [31], [36]):

- In the "decentral" mode, in fact, there is no coordination: each department autonomously makes the "own" partial decisions $\mathbf{d}_{t, r}^{\text {own }}$ and the overall configuration $\mathbf{d}_{t}$ of decisions results as a combination of these departmental choices without any central intervention. Hence, the function of the main office is limited to (perhaps inaccurately) 
observing the overall performance achieved.

- In a mode named "proposal" each department proposes two alternative configurations $\mathbf{d}_{t}$ to the main office and, among all proposals received, the main office finally chooses the one that promises the highest overall performance. Hence, by their proposals the departments shape the search space of the main office.

\section{B. Informational Structure}

Our agents identify superior solutions of the decisional problem according to the perceived contributions of the choices to their compensation or to overall performance, respectively. To represent inaccurate judgmental information which might be improved due the management accounting system in the course of the adaptive walk (Section III.C) - we add noise on the contributions of decisions to performance. Furthermore, in order to represent expertise related to segmentation and specialization we differentiate noise according to the information quality different decision-makers in an organization reasonably have. A common idea of many organizational theories is that decision-makers in organizations dispose of information with different levels of imperfections (e.g. [7], [8]). For example, departmental decision-makers are assumed to have relatively precise information about their own area of competence, but limited cross-departmental knowledge whereas the main office might have rather coarse-grained, but organization-wide information.

We assume that departments decide on basis of the perceived value base for compensation, i.e., the perceived overall performance rather than the actual. Therefore, we "distort" the actual performance contributions according to the expertise of each single department. In particular, the perceived value base for compensation, i.e., the overall performance $\tilde{V}_{t, r}\left(\mathbf{d}_{t}\right)$ department $r$ perceives, is computed as normalized sum of the actual own performance and actual residual performance, each distorted with an error term

$$
\tilde{V}_{t, r}\left(\mathbf{d}_{t}\right)=\left[\tilde{V}_{t, r}^{\text {own }}\left(\mathbf{d}_{t, r}^{\text {own }}\right)+\tilde{V}_{t, r}^{\text {res }}\left(\mathbf{d}_{t, r}^{\text {res }}\right)\right] / N
$$

where

$$
\begin{aligned}
& \tilde{V}_{t, r}^{\text {own }}\left(\mathbf{d}_{t, r}^{\text {own }}\right)=V_{t, r}^{\text {own }}\left(\mathbf{d}_{t, r}^{\text {own }}\right)+e_{r}^{\text {own }}\left(\mathbf{d}_{t, r}^{\text {own }}\right) \\
& \text { and } \tilde{V}_{t, r}^{\text {res }}\left(\mathbf{d}_{t, r}^{\text {res }}\right)=V_{t, r}^{\text {res }}\left(\mathbf{d}_{t, r}^{\text {res }}\right)+e_{r}^{\text {res }}\left(\mathbf{d}_{t, r}^{\text {res }}\right)
\end{aligned}
$$

Likewise, in the coordination mode "proposal" the main office makes a choice from the proposals on basis of the perceived overall performance $\tilde{V}_{t}\left(\mathbf{d}_{t}\right)$ computed as the sum of the true overall performance $V_{t}\left(\mathbf{d}_{t}\right)$ and an error term $e_{\text {main }}\left(\mathbf{d}_{t}\right)$.

At least with respect to accounting systems [20], it is reasonable to assume that high (low) true values of performance come along with high (low) distortions. Hence, we reflect distortions as relative errors imputed to the true performance (for other functions [35])., and, for simplicity, the error terms follow a Gaussian distribution $\mathrm{N}(\mu ; \sigma)$ with expected value $\mu=0$ and standard deviations $\sigma_{r}^{\text {own }}, \sigma_{r}^{\text {res }}$ and $\sigma_{\text {main }}$. For example, department $r$ perceives the "own" performance as

$$
\tilde{V}_{t, r}^{o w n}\left(\mathbf{d}_{t, r}^{\text {own }}\right)=V_{t, r}^{\text {own }}\left(\mathbf{d}_{t, r}^{\text {own }}\right) \cdot\left(1+N\left(0 ; \sigma_{r}^{\text {own }}\left(\mathbf{d}_{t, r}^{\text {own }}\right)\right)\right.
$$

We differentiate the standard deviations according to specialization of departments and the main office as mentioned above (see notes on parameter settings in Table 2).

\section{Basic Design Options of the Management Accounting Systems}

As argued in the introduction, within the search for higher levels of performance ex ante-evaluations might suffer from two deficiencies: The performance of the status quo option is misestimated and/or the performances of alternative options are inaccurately evaluated. This paper is particularly interested in the potentially beneficial role that measurements of the status quo by accounting systems can play for organizational performance. In our model, we therefore distinguish five settings of measurement and usage of actual values in the adaptive walk (summarized in Table 2) which may be regarded as basic design options of the management accounting system:

TABLE I

SETtings of MEASUREMENT AND Usage OF ACtUALS IN THE ADAPTIVE WALKS

\begin{tabular}{lcl}
\hline Name of Setting & $\begin{array}{l}\text { Measurement of } \\
\text { Actuals for Status Quo }\end{array}$ & $\begin{array}{l}\text { Adjustment of } \\
\text { Inaccuracies in } \\
\text { Adaptive Walk }\end{array}$ \\
\hline (1) No measurement & no & no \\
(2) Measurement only & yes & no \\
(3) Stepwise & yes & stepwise \\
(4) $\begin{array}{l}\text { Immedinement } \\
\text { adjustment }\end{array}$ & yes & immediately at \\
(5) Perfect evaluation & yes & once \\
(not necessary)
\end{tabular}

1) In case that "no measurement" is used the evaluation of the status quo configuration (i.e., the choice $\mathbf{d}_{i-1}^{*}$ made in period $t-1$ ) cannot be based on the measurement of the actual values achieved in the previous period. In a way, this reflects an organization which does not have any accounting system at all.

2) In a setting we call "measurement only" our departments use accounting systems which allow them to perfectly determine the performance that was achieved with the status quo configuration $\mathbf{d}_{i-1}^{*}$ of the decisional vector. Hence, throughout each adaptive walk, when department heads decide they perfectly get informed about the status quo by the accounting system. However, they suffer from inaccurate knowledge of the performance contributions of the alternative options $\mathbf{d}_{t, r}^{a 1}$ and $\mathbf{d}_{t, r}^{a 2}$, that they consider, i.e., the accounting system does not provide any refined information on the alternatives regardless of whether they have been implemented in the past or not. Thus, the accounting system does not provide any tracking or memory about the configurations that have been realized 
or any information for updating of beliefs (s. section II.A) on the alternative options.

3) A setting we name "stepwise refinement" goes a step further. Like in the previously described setting the decision-makers get perfect information about the performance of the status quo $\mathbf{d}_{i-1}^{*}$, and, additionally, the measured actual values are used for some kind of "learning". Hence, the management accounting system is used for updating of beliefs on alternative options according to [3]. For simplicity the stepwise refinement is represented in a relatively "mechanistic" form of noise reduction: whenever a certain configuration $\mathbf{d}$ of decisions has been implemented, decision-makers receive information about the related contributions to performance measures. This information will be partially memorized in future periods, and, in particular, will then lead to a refined estimation of performance of that configuration. This situation, for example, reflects a situation where cost functions applied for cost planning might be (even automatically) adjusted with each measurement of the performance that a certain configuration of cost drivers provides: with each determined combination of cost drivers and cost measures the statistical basis is broadened from which a cost function could be derived (for example by regression analysis). For each of the $n=2^{N}$ configurations $\mathbf{d}=\left(d_{1}, \ldots d_{\mathrm{N}}\right)$ in the solution space (due to $d_{i} \in\{0,1\}$ and $i=1, \ldots N)$ a counter count $^{n}$ is introduced. Whenever a certain configuration $\mathbf{d}^{*}$ is chosen/implemented during the observation period $T$ the related counter $\operatorname{count}^{d^{*}}$ of configuration $\mathbf{d}^{*}$ is incremented by 1 . Hence, if the performance contributions of this configuration $\mathbf{d}^{*}$ are evaluated again in a later period the corresponding errors $e_{r}^{\text {own }}, e_{r}^{r e s}$ and $e_{\text {main }}$ are divided by count $t^{d^{*}}$. Thus, for example, when under coordination mode "proposal" the main office again evaluates configuration $\mathbf{d}_{t}$ the main office perceives the overall performance as

$$
\tilde{V}\left(\mathbf{d}_{t}\right)=V\left(\mathbf{d}_{t}\right) \cdot\left(1+\frac{1}{\text { count }^{d_{t}}} \cdot e_{\text {main }}\left(\mathbf{d}_{t}\right)\right)
$$

4) The case "immediate adjustment" slightly differs from the "stepwise refinement" setting as the accounting systems provide perfect memorizing and immediate correction of ex ante-evaluations due to measured actual values for a configuration $\mathbf{d}^{*}$ that has been implemented. Hence, whenever in the adaptive walk a configuration is considered, which has already been implemented, at least once, during the walk, the decision-makers get perfect information about the level of performance as measured by the accounting system. For example, the main office evaluates the overall performance of a configuration $\mathbf{d}_{t}$ as

$$
\begin{aligned}
& \tilde{V}\left(\mathbf{d}_{t}\right)=V\left(\mathbf{d}_{t}\right) \cdot\left(1+e_{\text {main }}\left(\mathbf{d}_{t}\right)\right) \text { for } \text { count }^{d_{t}}=1 \\
& \tilde{V}\left(\mathbf{d}_{t}\right)=V\left(\mathbf{d}_{t}\right) \text { for } \text { count }^{d_{t}} \geq 2
\end{aligned}
$$

5) Perfect evaluations in our simulations serve as a "benchmark" so that performance differences due to imperfect evaluations can be determined. Here neither the evaluations of the status quo $\mathbf{d}_{i-1}^{*}$ nor of the alternative options $\mathbf{d}_{t, r}^{a 1}$ or $\mathbf{d}_{t, r}^{a 2}$ suffer from any noise, i.e., all error terms are set to zero.

\section{RESULTS AND INTERPRETATION}

\section{A. Parameter Settings in the Simulation Experiments and Measures for Effectiveness}

For simulating an adaptive walk, after a "true" fitness landscape is generated, distortions are added which follow the informational imperfections in the organization as described in section III.B. Then the organizations are placed randomly in the fitness landscape and observed for 300 periods while searching for higher levels of organizational performance under the regime of various settings of management accounting systems as introduced in section III.C. As is familiar for adaptive walks we use a hill-climbing algorithm. In particular, each decision maker evaluates the options he/she knows (i.e., status quo and alternatives) and an alteration is preferred in favor of that option which promises the steepest ascent.

The results were conducted for two interaction structures of decisions (i.e., coordination needs) which, in a way, represent two extremes (for these and other interaction structures see [32]): in the low complexity case intra-departmental interactions among decisions are maximal intense while no cross-departmental interdependencies exist. This type of interactions corresponds to a "self-contained" organization structure [7] and comes close to a pooled interdependence [37], [38]. In contrast, in the high complexity case all decisions affect the performance contributions of all other decisions, i.e., the complexity of interactions and the coordination need is raised to maximum. This situation comes closest to a reciprocal interdependence [37], [38].

Empirical findings report errors of judgmental information between 5 up to 30 percent [39], [40]. Results presented in this paper relate to errors around 10 percent though differentiated due to specialization as described in section III.B and as explicated in the note to Table 2. (It is worth mentioning, that results were subject to robustness analyses, especially with respect to the magnitude of errors and the spread between knowledge about the "own" area of competence and the rest of the organization. We found that the results appear robust in a range up to a magnitude of overall error around 22 percent and with several levels of spread according to specialization of decision-makers.)

For investigating the effectiveness of the adaptive walks we rely on three measures as displayed in Table 2: "Speed $\left(V_{5}-V_{1}\right)$ " reports the performance enhancements achieved in the first 5 periods within the adaptive walks. This measure appears interesting because in the first periods most purely the 
International Journal of Artificial Intelligence and Interactive Multimedia, Vol. 2, $N^{\circ} 4$.

TABLE II

CONDENSED RESULTS

\begin{tabular}{lrrrrrr}
\hline & Low Complexity & \multicolumn{3}{c}{ High Complexity } & \\
Name of Setting & $\begin{array}{r}\text { Speed } \\
\left(V_{5}-V_{1}\right)\end{array}$ & $\begin{array}{r}\text { Performal } \\
\left(V_{300}\right)\end{array}$ & $\begin{array}{r}\text { Frequency of } \\
\text { Global } \\
\text { Maximum in } \\
t=300\end{array}$ & $\begin{array}{r}\text { Speed } \\
\left(V_{5}-V_{1}\right)\end{array}$ & $\begin{array}{r}\text { Performance } \\
\left(V_{300}\right)\end{array}$ & $\begin{array}{r}\text { Frequency of } \\
\text { Global } \\
\text { Mum in } \\
t=300\end{array}$ \\
\hline Decentral Mode & & & & & & \\
(1) No measurement & 0.04419 & 0.83689 & $1.66 \%$ & 0.12479 & 0.84374 & $1.94 \%$ \\
(2) Measurement only & 0.05211 & 0.85251 & $1.94 \%$ & 0.12488 & 0.86941 & $3.16 \%$ \\
(3) Stepwise refinement & 0.05734 & 0.89748 & $8.24 \%$ & 0.12818 & 0.86738 & $3.00 \%$ \\
(4) Immediate adjustment & 0.05771 & 0.89381 & $7.76 \%$ & 0.12121 & 0.86519 & $3.46 \%$ \\
(5) Perfect evaluation & 0.07321 & 0.89730 & $10.16 \%$ & 0.14536 & 0.86466 & $2.44 \%$ \\
Proposal Mode & & & & & & \\
(1) No measurement & 0.06878 & 0.83506 & $1.46 \%$ & 0.05739 & 0.83541 & $1.76 \%$ \\
(2) Measurement only & 0.07277 & 0.85222 & $2.52 \%$ & 0.05754 & 0.83665 & $1.90 \%$ \\
(3) Stepwise refinement & 0.08473 & 0.87681 & $4.84 \%$ & 0.06387 & 0.85041 & $2.30 \%$ \\
(4) Immediate adjustment & 0.08601 & 0.87724 & $4.56 \%$ & 0.06742 & 0.84803 & $1.78 \%$ \\
(5) Perfect evaluation & 0.09781 & 0.89518 & $9.54 \%$ & 0.06510 & 0.86716 & $2.40 \%$
\end{tabular}

Notes: Each entry represents results of 5,000 adaptive walks: 1,000 distinct fitness landscapes with 5 adaptive walks on each over 300 periods. Confidence intervals for $V_{300}$ at a confidence level of 0.001 range between \pm 0.003 and \pm 0.004 . Common parameters in settings (1) to (4): $\sigma_{r}^{\text {own }}=0.05, \sigma_{r}^{\text {res }}=0.15$ and $\sigma_{\text {main }}=0.1$ (in (5) all set to 0); all errors with expected value $\mu=0$.

effects of refinements (settings 3 and 4 ) can be observed. The performance in the last observation period $V_{300}$ can serve as an indicator for the effectiveness of the search process as well as the frequency of how often the global maximum in the performance landscape is achieved in the last period observed.

Furthermore, Fig. 1 and Fig. 2 reflect the performance differences in the course of the adaptive walks of the noisy against the perfect evaluations for low and high complexity of cross-departmental interactions.

We discuss results in two steps. Firstly, we focus on comparing the different settings of measuring and using actuals against each other (Section IV.B) and afterwards we discuss the moderating effects of complexity and coordination (Section IV.C).

\section{B. Effectiveness of Various Settings of Management Accounting Systems}

Obviously, evaluating alternative options with imperfect information can result in a choice which appears favorable, whereas, in fact, it reduces performance compared to the status quo ("false positive" decision) [35]. Underestimating the status quo level of performance due to missing or imperfect measurement of actuals might foster the false estimation. Vice versa, with "false negative" decisions an alternative is rejected because its marginal contribution to performance compared to the status quo appears worse than it actually is and, thus, the status quo is perpetuated [35]. This situation may be fostered by an overestimation of the status quo level of performance. These considerations let us hypothesize the following:

With increasing levels of measurement and usage of actual values for improving judgmental information (1) the speed of performance enhancements increases and (2) higher levels of organizational performance are achieved.

The five settings of management accounting systems in terms of measuring actual values and using these numbers for judgments as displayed in table 1 incorporate an order of increasing information accuracy. We find that the speed measure $\left(V_{5}-V_{1}\right)$ in Table 2 in most cases is increasing with the more advanced settings of accounting systems. Furthermore, as Fig. 1 and Fig. 2 show at a glance, the more advanced settings of management accounting systems tend to have lower performance losses against the perfect system. However, the results provide broad, but no universal support for the hypothesis stated above and some of the results deserve a closer analysis.

First of all, it is worth mentioning that under setting (1) where no actual numbers are available at all, the performance achieved is lowest in all of the four scenarios of coordination need and mode -- in three scenarios with remarkable performance losses even to the "measurement only" setting (we discuss the "high complexity-proposal mode"-scenario below more into detail). Apparently, over- or underestimating the status quo leads to severe losses of speed and level of performance enhancements. Hence, this indicates that using an accounting system, at least, to track the status quo (e.g., an actual cost system) is effective.

Secondly, the results for the "stepwise refinement" and the "immediate adjustment" are rather similar for all scenarios under investigation. An obvious reason is that the "stepwise refinement" setting is modeled in a way that the decisionmakers get better knowledge of the fitness landscape relatively fast. The simulation of a slower learning curve might yield other results.

Thirdly, "stepwise refinement" and "immediate adjustment" of knowledge about the fitness landscape bring performance to levels higher than achieved with "measurement only" -- except for the case of high complexity and decentral coordination which is discussed in section IV.C. Obviously, it is less likely 

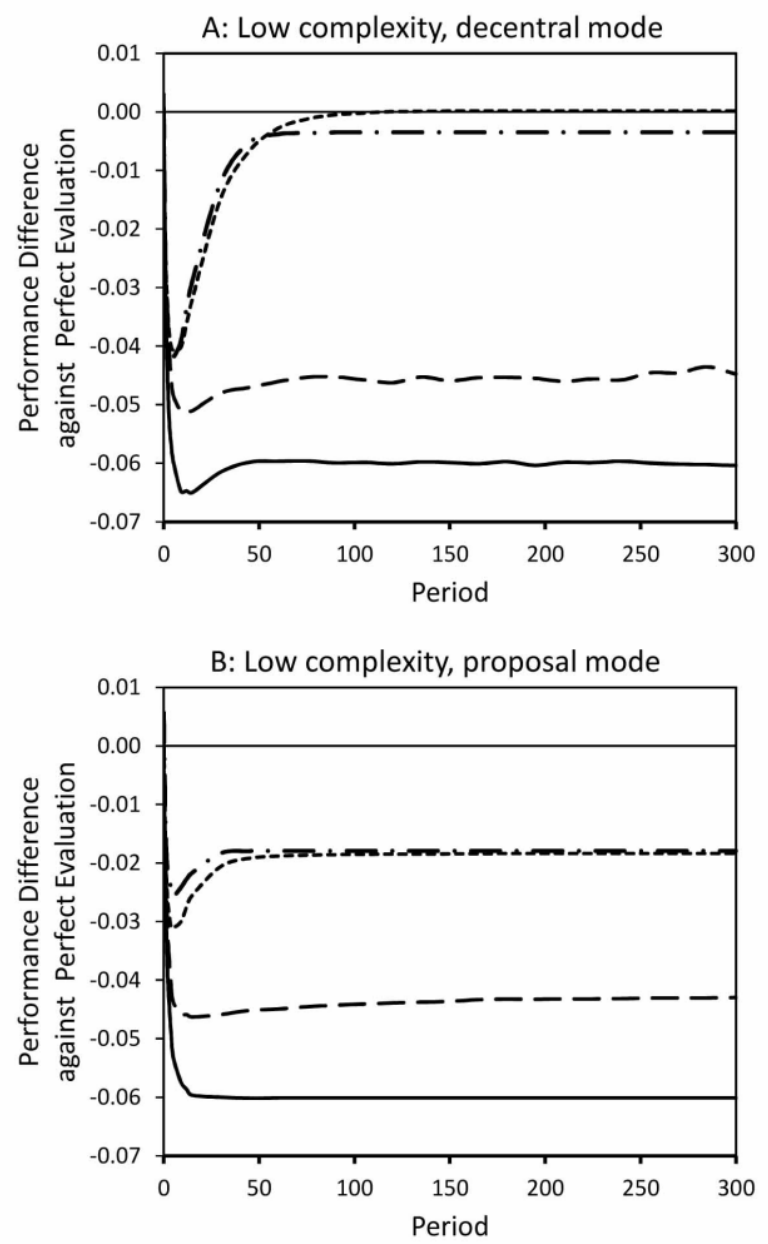

with

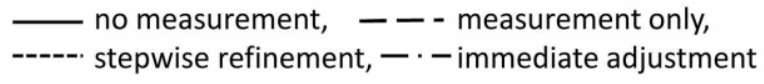

Fig. 1. Performance differences against perfect evaluations in case of no cross-departmental interactions

Notes: The horizontal line at level 0 of the $y$-axis reflects the perfect evaluation and the other lines represent the performance differences in the course of the adaptive walks against the perfect management accounting system. Each line represents results of 5,000 adaptive walks: 1,000 distinct fitness landscapes with 5 adaptive walks on each over 300 periods. For parameter settings see Table 1 and notes to Table 2.

to opt for a false positive or false negative alternative within the adaptive walks in case of the immediate or stepwise improvement of judgmental information by actuals. This indicates that accounting systems which allow memorizing actual values contribute to higher performance levels.

\section{Effects of complexity and coordination mode}

The results provide broad support for intuition that increasing accuracy of the management accounting systems captured in settings 1 to 5 leads to faster performance enhancements und higher levels of final performance. However, some results run contrary to intuition. In particular, the complexity of the interactions structure (i.e., coordination need) and the coordination mode applied apparently interfere with the information accuracy provided by the management accounting system.

We start the discussion of the effects of coordination need and mode with the scenario of "low complexity-decentral
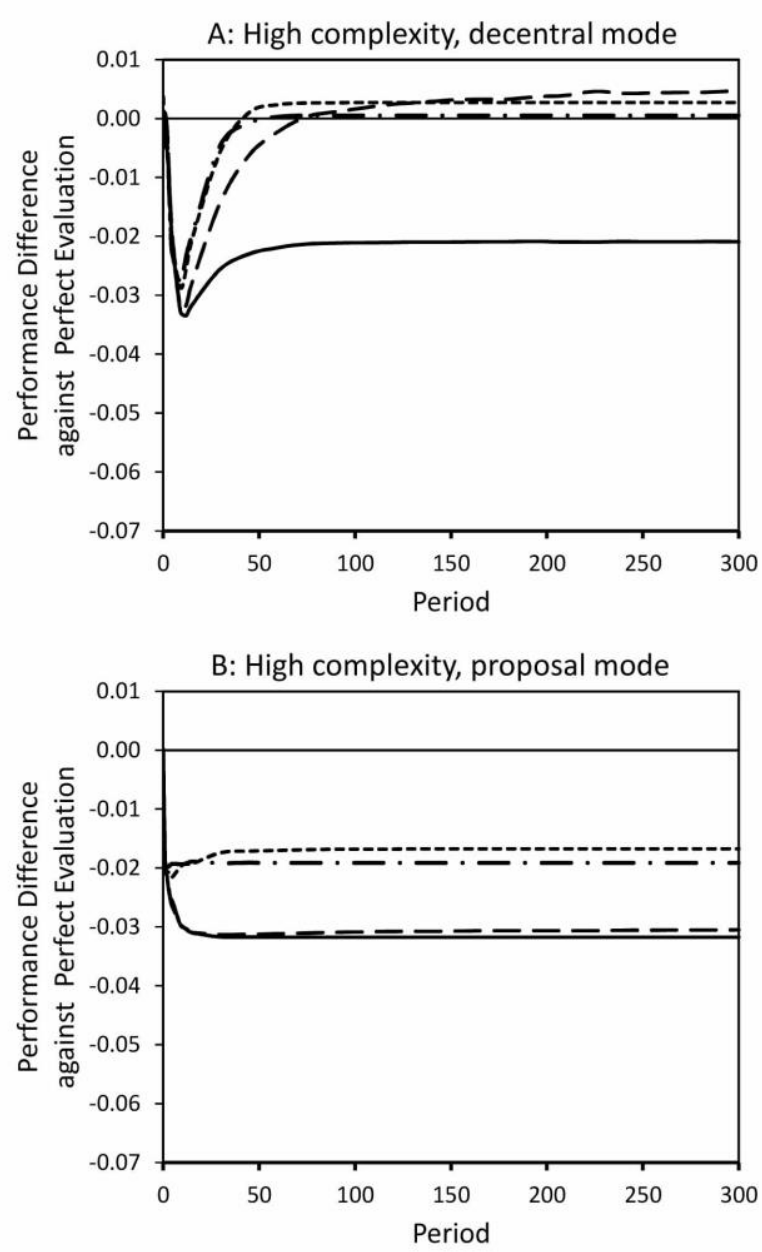

with

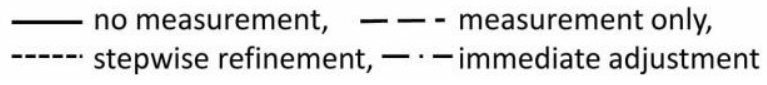

Fig. 2. Performance differences against perfect evaluations in case of maximum cross-departmental interactions

Notes: The horizontal line at level 0 of the y-axis reflects the perfect evaluation and the other lines represent the performance differences in the course of the adaptive walks against the perfect management accounting system. Each line represents results of 5,000 adaptive walks: 1,000 distinct fitness landscapes with 5 adaptive walks on each over 300 periods. For parameter settings see Table 1 and notes to Table 2.

mode" (Fig. 1A). In this case no cross-departmental interactions exist. Therefore, no cross-departmental coordination is required: with imperfect judgmental information departments might decide in favor of a suboptimal partial option (false positive or false negative), but there are no external effects in the sense that this would reduce the performance of the other departments' decisions. The accounting systems 3 and 4, after around 75 periods in average reach the level of perfect information while systems 1 and 2 induce a rather high, nearly constant distance to perfect evaluations.

To a certain extent, things seem to change for highly intense cross-departmental interactions among decisions (Fig. 2A). In particular, with decentral coordination for high complexity even the "measurement only" setting leads to performance levels beyond that achieved with perfect evaluations. Fig. 2A indicates that after around 50 to 75 periods the noisy 
accounting systems with measurements of actuals (i.e. settings (2), (3) and (4) in table 1) exceed the performance achieved with perfect evaluations.

In order to provide an explanation for this "beneficial" effect of noise we refer to "false positive" evaluations. Of course, with "false positives" an organization goes a "wrong way" for a short term, but with the chance to discover superior configurations in a longer term [15], [34]. In particular, imperfect knowledge may afford the opportunity to leave a local peak in the fitness landscape. We argue that this effect is the more likely the more interactions among decisions exist: as is well investigated for the NK model in literature (e.g. [29], [30]), with higher levels of complexity the more rugged is the fitness landscapes and the more local maxima exist, and, hence, the search process is more likely to stick to a local maximum. Inaccuracies induce diversity in the search process, and "false positive" alterations, though short-term harmful, provide the chance to discover superior levels of performance and, eventually, the global maximum in the long-term. The results provide support for this intuition: In the "high complexity-decentral mode" scenario the relative frequency of how often the global maximum is found is lower with perfect evaluations than with noisy accounting systems as far as they measure the status quo.

In the next step we analyze the role of the coordination mode. Firstly, results indicate that with the proposal mode (i.e., with involving the main office in decision-making) the range of differences in speed and level of performance among the various forms of management accounting systems. Hence, in a way, with introducing the information-processing power of the main office the relevance of the setting of the accounting system tends to be reduced.

Secondly, our results (Fig. 1A versus 1B and Fig. 2A versus 2B) also suggest that with inaccurate judgmental information in the proposal mode organizations miss the chance to achieve those performance levels that can be reached with perfect evaluations. In order to provide an explanation we find it helpful to remember that in the proposal mode the status quo only is abandoned if two conditions are met. First, at least, one department has to discover a partial vector that promises a higher compensation to the respective department head (otherwise he/she would not propose the alteration); second, the main office has to accept the proposal. Hence, for being implemented each proposal has to pass an additional instance and, hence, it is less likely that false positive evaluations on the departments' site affect final decisions since the main office may detect the false positive evaluations [22].

However, by that, the "false positives" are less likely to do their beneficial work as discussed above. Furthermore, "false negative" evaluations by the main office might occur and the organization is more likely to suffer from inertia compared to the decentral mode. With more inertia the fitness landscape is less likely to be "explored" and this reduces benefits of the "stepwise refinement" and "immediate adjustment" accounting systems: To enfold the full potential of "learning" management accounting systems (settings 3 and 4) a certain exploration of the decisional space is required, which apparently might not be given in the proposal mode.

\section{V.CONCLUSION}

The results provide broad support for the intuition that improving judgmental information by measurements of actual values in management accounting systems leads to more effective adaptive search processes for higher levels of organizational performance.

However, the results might throw some new light on basic design choices of management accounting systems: apparently, the contribution of improving information accuracy in management accounting systems subtly interferes with coordination need and mode. In particular, results do not universally support conventional wisdom that better accountting systems are more beneficial when decision-problems are highly complex. Furthermore, our results suggest that inaccuracies might have their positive sides compared to perfect information for complex decisions - given that inaccuracies are accompanied by decentral coordination.

Moreover, it appears that with more central coordination the relevance of improving information quality in the management accounting system decreased. In short, to a certain extent management accounting systems and central coordination power seem to serve as substitutes. Hence, taking into account that improvements of management accounting systems usually are not costless, these findings put claims for investments in perspective.

At the same time, our analysis is subject to several limitations which should be overcome in further research. First of all, it should be mentioned that in our model the contributions of management accounting systems to ex anteevaluations of alternatives is represented in a rather coarse way. Of course, more sophisticated learning and forecasting methods could be integrated (i.e., methods applied in plan cost accounting systems). Moreover, the ex ante-evaluations of our decision-makers suffer from imperfect knowledge about the "production functions" (in terms of the relation between choice and organizational outcome), but the model presented does neither reflect conflicts of interests nor decision-making under uncertainty. Obvious extensions of the model could overcome these shortages, especially in order to address the function of management accounting systems to update beliefs of decisionmakers as elaborated by Christensen [3].

Furthermore, organizations apply various strategies and coordination modes to deal with imperfectly known "production functions". In further extensions these strategies could be reflected in the model as well as the decision-making biases (e.g., status-quo bias) that decision-makers suffer from [11]. Including these aspects could reveal further insights into the relative benefits of basic design options of management accounting systems. 


\section{REFERENCES}

[1] J. S. Demski, and G. A. Feltham, Cost Determination: A Conceptual Approach. Ames: Iowa State Univ. Press, 1976.

[2] C. T. Horngren, S. M. Datar, G. Foster, M. Rajan, and C. Ittner, Cost Accounting: A Managerial Emphasis. New York: Prentice Hall, 2005.

[3] J. Christensen, "Accounting Errors and Errors of Accounting," The Accounting Review, vol. 85, no. 6, pp. 1827-1838, 2010.

[4] H. A. Simon, "A Behavioral Model of Rational Choice," Quarterly Journal of Economics, vol. 69, no. 1, pp. 99-118, Feb. 1955.

[5] C. T. Horngren, A. Bhimani, S. M. Datar, and G. Foster, Management and Cost Accounting. 3. ed., Essex: Pearson, 2005.

[6] J. Marschak, "Towards an Economic Theory of Organization and Information." in Decision Processes, R. M. Thrall, C. Coombs, and R.L. Davis, Eds. New York: Wiley, 1954, pp. 187--220.

[7] J. Galbraith, Designing Complex Organisations. Reading: AddisonWesley, 1973.

[8] M. J. Ginzberg, "An Organizational Contingencies View of Accounting and Information Systems Implementation," Accounting, Organizations and Society, vol. 5, pp. 369-382, 1980.

[9] R. L. Ackoff, "Management Misinformation Systems," Management Science, vol. 14, no. 4, B-147-B-156, 1967.

[10] R. L. Keeney, "Decision Analysis," Operations Research, vol. 30, no. 5, pp. 803-838, 1982.

[11] A. Tversky, and D. Kahneman, "Judgment under Uncertainty: Heuristics and Biases," Science, vol. 185, no. 4157, pp. 1124-1131, Sept. 1974.

[12] R. M. Bushman, and R. J. Indjejikian, "Aggregate Performance Measures in Business Unit Manager Compensation: The Role of Intrafirm Interdependencies," Journal of Accounting Research, vol. 33 , supplement, pp. 101-129, 1995.

[13] R. P. Brief, "Accounting Error as a Factor in Business History," Accounting, Business and Financial History, vol. 1, pp. 7-21, 1990.

[14] J. P. Davis, K. M. Eisenhardt, and C. B. Bingham, "Developing theory through simulation methods," Academy of Management Review, vol. 32, no. 2, pp. 480-499, 2007.

[15] F. Wall, "The (Beneficial) Role of Informational Imperfections in Enhancing Organisational Performance," in Progress in Artificial Economics, M. LiCalzi, L. Milone, and P. Pellizzari, Eds. Berlin: Springer-Verlag, 2010, pp. 115-126.

[16] S. Leitner, "Interactions among Biases in Costing Systems: A Simulation Approach," in Managing Market Complexity. The Approach for Artificial Economics. S. Alfarano, A. Teglio, E. Camacho-Cuena, and M. Ginés-Vilar, Eds., Heidelberg: Springer-Verlag, 2012, pp. 209-220.

[17] S. Leitner, "A Simulation Analysis of Interactions among Intended Biases in Costing Systems and their Effects on the Accuracy of Decision-Influencing Information (Accepted for publication)," Central European Journal of Operations Research, DOI: 10.1007/s10100-0120275-2, online-first since Nov. 2012.

[18] F. Wall, "Comparing Basic Design Options for Management Accounting Systems with an Agent-Based Simulation," in Distributed Computing and Artificial Intelligence, S. Omatu, J. Neves, J. M. C. Rodriguez, J.F. Paz Santana, and S.R. Gonzalez, Eds. Heidelberg: Springer-Verlag, 2013, pp. 409-418.

[19] S. Datar, and M. Gupta, "Aggregation, Specification and Measurement Error in Product Costing," The Accounting Review, vol. 69, no. 4, pp. 567-591, Oct. 1994.

[20] E. Labro, and M. Vanhoucke, "A Simulation Analysis of Interactions among Errors in Costing Systems," The Accounting Review, vol. 82, no. 4, pp. 939-962, July 2007.

[21] R. K. Sah, and J. E. Stiglitz, "Human Fallibility and Economic Organization," American Economic Review, vol. 75, no. 2, pp. 292-297, 1985.

[22] R. K. Sah, and J. E. Stiglitz, "The Architecture of Economic Systems: Hierarchies and Polyarchies," American Economic Review, vol. 76, no. 4, pp. 716-727, 1986.

[23] R. K. Sah, and J. E. Stiglitz, "Committees, Hierarchies and Polyarchies," The Economic Journal, vol. 98, pp. 451-470, June 1988.

[24] W. T. H. Koh, "Human Fallibility and Sequential Decision Making: Hierarchy versus Polyarchy" Journal of Economic Behavior and Organization, vol. 18, no. 3, pp. 317-345, 1992.
[25] W. T. H. Koh," Making Decisions in Committees: A Human Fallibility Approach," Journal of Economic Behavior and Organization, vol. 23, no. 2, pp. 195-214, 1994.

[26] B. Visser, "Organizational communication structure and performance," Journal of Economic Behavior and Organization, vol. 42, no. 2, pp. 231-252, 2000

[27] M. Christensen, and T. Knudsen, "Design of decision-making organizations," Management Science, vol. 56, no. 1, pp. 71-89, Jan. 2010.

[28] S. A. Kauffman, The Origins of Order: Self-Organization and Selection in Evolution. Oxford: Oxford Univ. Press, 1993.

[29] S. A. Kauffman, and S. Levin, "Towards a General Theory of Adaptive Walks on Rugged Landscapes," Journal of Theoretical Biology, vol. 128, no. 1, pp. 11-45, Sept. 1987.

[30] J. W. Rivkin, and N. Siggelkow, "Balancing Search and Stability: Interdependencies among Elements of Organizational Design," Management Science, vol. 49, no. 3, pp. 290-311, March 2003.

[31] N. Siggelkow, and J. W. Rivkin, "Speed and Search: Designing Organizations for Turbulence and Complexity," Organization Science, vol. 16, no. 2, pp. 101-122, March-April 2005.

[32] Rivkin, J.W., Siggelkow, N.: Patterned Interactions in Complex Systems: Implications for Exploration. Management Science 53, 1068-1085 (2007)

[33] M. Chang, and J. E. Harrington, "Agent-Based Models of Organizations" in Handbook of Computational Economics, vol. 2, L. Tesfatsion, and K. L. Judd, Eds. Amsterdam: Elsevier 2005, pp. 1273-1337.

[34] T. Knudsen, and D. A. Levinthal, "Two Faces of Search: Alternative Generation and Alternative Evaluation," Organization Science, vol. 18, no. 1, pp. 39-54, Jan.-Feb. 2007.

[35] B. Levitan, and S. A. Kauffman, "Adaptive Walks with Noisy Fitness Measurements," Molecular Diversity, vol. 1, no. 1, pp. 53-68, Sept. 1995.

[36] G. Dosi, D. Levinthal, and L. Marengo. L, "Bridging Contested Terrain: Linking Incentive-based and Learning Perspectives on Organizational Evolution," Industrial and Corporate Change, vol. 12, no. 2, pp. 413-436, 2003.

[37] T. W. Malone, and K. Crowstone, "The interdisciplinary study of coordination," ACM Computing Surveys, vol. 26, no. 1, pp. 87-119, 1994.

[38] J. D. Thompson. Organizations in action. Social science bases of administrative theory. New York: McGraw-Hill 1967.

[39] S. W. Tee, and P. L. Bowen, P. Doyle, and F. H. Rohde, "Factors Influencing Organizations to Improve Data Quality in their Information Systems," Accounting and Finance, vol. 47, no. 2, pp. 335-355, 2007.

[40] T. C. Redman, Data Quality for the Information Age. Boston/London: Artech House, 1996.

Friederike Wall earned her Diploma for Business Economics in 1988 and her Doctoral degree in 1991, both at the Georg-August-Univesität Göttingen, Germany. In 1996 she received the "venia legendi" (Habilitation for Business Economics) from the Universität Hamburg, Germany. After being a scientific Project Referent for Accounting in the context of the implementation of SAP R/3 at Max-Planck Society, Munich, Germany she became Full Professor of Business Administration, esp. Controlling and Information Management at the Universität Witten/Herdecke, Germany. Since 2009 she is Full Professor and Head of the Department of Controlling and Strategic Management at the Alpen-Adria-Universität Klagenfurt, Austria. Prof. Wall's scientific work is focused on management accounting systems and the quality of the information provided by these systems. Her main research approach is defined by agent-based simulation methods and agent-based technologies. 